\title{
Sore throat progressing to embolic sepsis: A case of Lemierre's syndrome
}

\author{
Jacquelyn Dirks MD, Dennis Bowie MD FRCPC
}

\begin{abstract}
J Dirks, D Bowie. Sore throat progressing to embolic sepsis: A case of Lemierre's syndrome. Can Respir J 2010;17(1):e20-e22.

Lemierre's syndrome is a rare clinical syndrome defined as oropharyngeal sepsis, thrombophlebitis of the internal jugular vein and septic thomboemboli. It is typically encountered in young, immunocompetent individuals, with a mean incident age of 20 years. The organism that is most commonly associated is an anaerobic Gram-negative bacterium: Fusobacterium species. The defined treatment course is at least six weeks of antibiotics, with the role of anticoagulation being unclear. The present article documents a case of Lemierre's syndrome complicated by acute renal failure and loculated pleural effusion in an otherwise healthy 16 -year-old patient.
\end{abstract}

Key Words: Fusobacterium; Lemierre's syndrome; Septic thrombophlebitis

A 16-year-old African Canadian man, who was otherwise A healthy, presented to the emergency room (ER) with a seven-day history of sore throat. The patient's symptoms began as dysphagia and progressed over six days to include a lowgrade fever, shortness of breath on exertion and inspiratory chest pain. He had seen his family doctor on day 2 with flu-like symptoms and received a five-day course of azithromycin, which did not help his symptoms. He felt unwell enough to visit the ER on day 3 of his illness. ER records indicated that he appeared to be a healthy 16-year-old boy complaining of moderate dysphagia and mild inspiratory chest pain. His vitals were stable but his temperature was $39.5^{\circ} \mathrm{C}$. He was advised to finish his course of antibiotics and to continue treating symptoms. Unfortunately, his inspiratory chest pain did not improve and his fever continued; consequently, he returned to the ER on day 7 of his illness.

On assessment in the ER, the patient described his chest pain as worse with inspiration, sharp in nature and localized to the lower left chest. The pain began on day 3 and worsened during the subsequent four days, and did not change with position. His shortness of breath worsened over three days and persisted at rest. His sore throat improved on days 5 and 6 , and became very mild. He did not complain of neck pain or stiffness. He was mildly nauseated; however, he had not vomited. He had had several loose stools over the previous three days all nonbloody.

Before the illness, the patient had been well. He was healthy throughout his childhood and his vaccinations were up to date. His developmental history was normal and he was a good student attending grade 11 . He was a nonsmoker, did not use alcohol or any illicit drugs, and was not sexually active. He had completed his azithromycin prescription and had been taking acetaminophen $975 \mathrm{mg}$ every $6 \mathrm{~h}$, as well as ibuprofen $600 \mathrm{mg}$ every $4 \mathrm{~h}$ for the previous two days.

On examination, the patient appeared acutely unwell. His heart rate was 130 beats/min and regular, with a blood pressure

\section{Maux de gorge progressant jusqu'à l'embolie septique : Cas de syndrome de Lemierre}

Le syndrome de Lemierre est un rare syndrome clinique caractérisé par une infection oropharyngée, la thrombophlébite de la veine jugulaire interne et la thromboembolie septique. On la rencontre généralement chez de jeunes sujets immunocompétents dont l'âge moyen est de 20 ans. L'agent le plus généralement associé est une bactérie anaérobie à Gram négatif, le genre Fusobacterium. Le traitement consiste en l'administration d'une antibiothérapie d'une durée minimum de six semaines et le rôle de l'anticoagulation reste à élucider. Le présent article illustre un cas de syndrome de Lemierre compliqué d'une insuffisance rénale aiguë et d'un épanchement pleural loculé chez un jeune homme de 16 ans par ailleurs en bonne santé.

of $106 / 60 \mathrm{mmHg}$ and a repiratory rate of 35 breaths/min. His tissue oximetry was $94 \%$ on $4 \mathrm{~L}$ of oxygen via nasal prongs and his temperature was $38.7^{\circ} \mathrm{C}$. His mucous membranes were dry and he was complaining of thirst. He had full range of neck motion and did not complain of stiffness. There were decreased breath sounds to the left lung base, with inspiratory crackles on the left. The abdomen was not tender and his spleen was not palpable. The remainder of the examination was normal.

The patient's admission laboratory results revealed a white blood count of $17.9 \times 10^{9} / \mathrm{L}$, with a neutrophilic shift. Hemoglobin was $100 \mathrm{~g} / \mathrm{L}$, with a platelet count of $37 \times 10^{9} / \mathrm{L}$ (normal $150 \times 10^{9} / \mathrm{L}$ to $350 \times 10^{9} / \mathrm{L}$ ). His urea measured $26.4 \mathrm{mmol} / \mathrm{L}$ (normal $2.9 \mathrm{mmol} / \mathrm{L}$ to $9.3 \mathrm{mmol} / \mathrm{L}$ ), with a serum creatinine of $332 \mu \mathrm{mol} / \mathrm{L}$ (normal $35 \mu \mathrm{mol} / \mathrm{L}$ to $88 \mu \mathrm{mol} / \mathrm{L}$ ). A chest $\mathrm{x}$-ray was performed and reported as airspace disease in the lower lobes, bilaterally consistent with pneumonia.

The patient was admitted to the intensive care unit (ICU) and started on $50 \%$ oxygen via a Venturi facemask. He was aggressively volume-resuscitated with normal saline. Blood was drawn for cultures and the patient received a dose of piperacillin/tazobactam. No central lines were placed because the patient had good peripheral access. A follow-up chest $\mathrm{x}$-ray was performed $5 \mathrm{~h}$ after admission (Figure 1) and was reported as showing worsening of the bilateral airspace disease.

The patient achieved a urine output of $50 \mathrm{~mL} / \mathrm{h}$ to $60 \mathrm{~mL} / \mathrm{h}$ with a $2 \mathrm{~L}$ bolus of normal saline, followed by $200 \mathrm{~mL} / \mathrm{h}$ maintenance. The creatinine level fell to $250 \mu \mathrm{mol} / \mathrm{L}$ within $8 \mathrm{~h}$ of volume resuscitation. Given the severity of his illness and low platelet count, a disseminated intravascular coagulation screen was performed. His international normalized ratio was 1.2, fibrinogen at $4.1 \mu \mathrm{mol} / \mathrm{L}$ and partial thromboplastin time $24 \mathrm{~s}-$ all within normal limits. A D-dimer was elevated at $3371 \mathrm{ng} / \mathrm{L}$. No schistocytes were seen on peripheral smear. A sickling hemoglobin screen was negative. Serum immunoglobulin M levels for Epstein-Barr virus were negative. A throat swab was negative for Streptococcal species.

Dalhousie University, Halifax, Nova Scotia

Correspondence: Dr Jacquelyn Dirks, Divison of Respirology, Dalhousie University, Department of Internal Medicine, 4479 Halifax Infirmary,

1796 Summer Street, Halifax, Nova Scotia B3H 3A7. Telephone 902-473-6611, fax 902-473-6202, e-mail dirks.jackie@gmail.com 


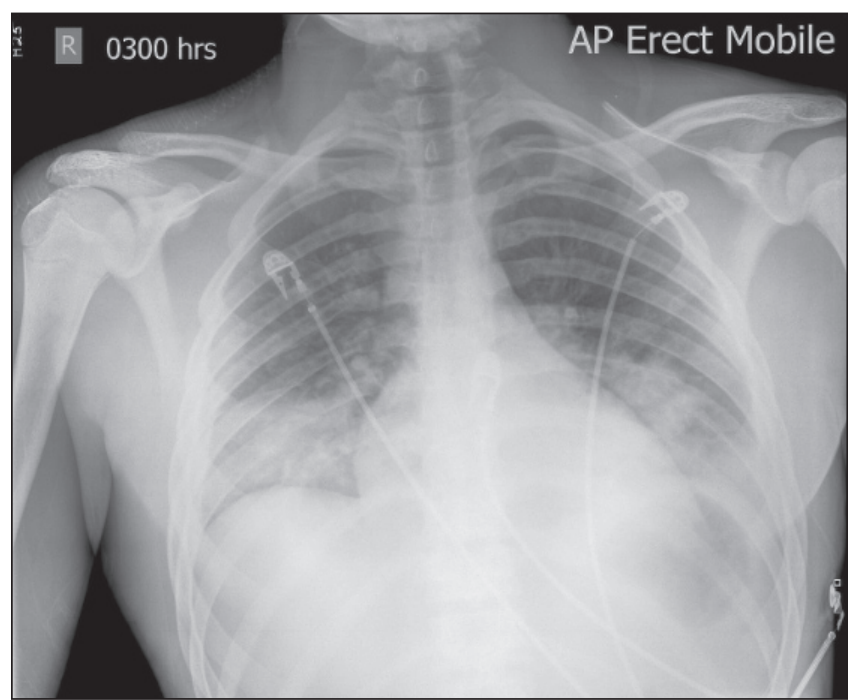

Figure 1) Chest radiograph showing bilateral septic thromboemboli

Sputum and urine cultures were negative after five days, with no growth. Blood cultures were positive after $24 \mathrm{~h}$ of incubation from the anaerobic bottle only. The organism was identified as a Gram-negative rod. The patient's antibiotics were switched to levofloxacin and metronidazole. The laboratory had difficulty identifying the organism and the genus was not available for four days. The patient was maintained on $50 \%$ oxygen via facemask for $48 \mathrm{~h}$ and was gradually weaned to room air over a four-day period. His urine output remained greater than $50 \mathrm{~mL} / \mathrm{h}$ throughout admission, with a high rate of intravenous maintenance. His serum creatinine fell to $81 \mu \mathrm{mol} / \mathrm{L}$ over four days. His platelet count rose steadily throughout admission and on day 5 was $107 \times 10^{9} / \mathrm{L}$. After five days in hospital, the patient's vital signs had stabilized and he no longer required oxygen. He was discharged directly from the ICU with a prescription for oral levofloxacin and metronidazole. On the day of discharge, the blood culture identified Fusobacterium species as the genus of the organism.

The patient was seen as an outpatient 10 days after discharge from the ICU. He had been afebrile since discharge and was regaining his appetite. However, he continued to complain of mild left-sided inspiratory chest pain, for which a follow-up chest $\mathrm{x}$-ray was performed (Figure 2) and revealed a loculated effusion that was tapped under ultrasound guidance. Chemistry revealed a sterile exudative effusion with a $\mathrm{pH}$ of 7.78 . This was left to reabsorb naturally. Given that the patient's microbiology and constellation of symptoms were consistent with Lemierre's syndrome, an in-clinic diagnostic ultrasound was performed, looking for evidence of internal jugular vein (IJV) thrombosis. The left jugular vein was patent. A nonocclusive clot was seen in the proximal IJV some two weeks after presentation (Figure 3). The patient was given a prescription for metronidazole for a total of eight weeks. Anticoagulation was not initiated. The patient was sent home with plans for a second $\mathrm{x}$-ray to ensure resolution of the pleural effusion, as well as follow-up with an infectious disease specialist. Three weeks after stopping all antibiotics, he was asymptomatic, with no fever or chest pain, and his x-ray showed no pleural effusion.

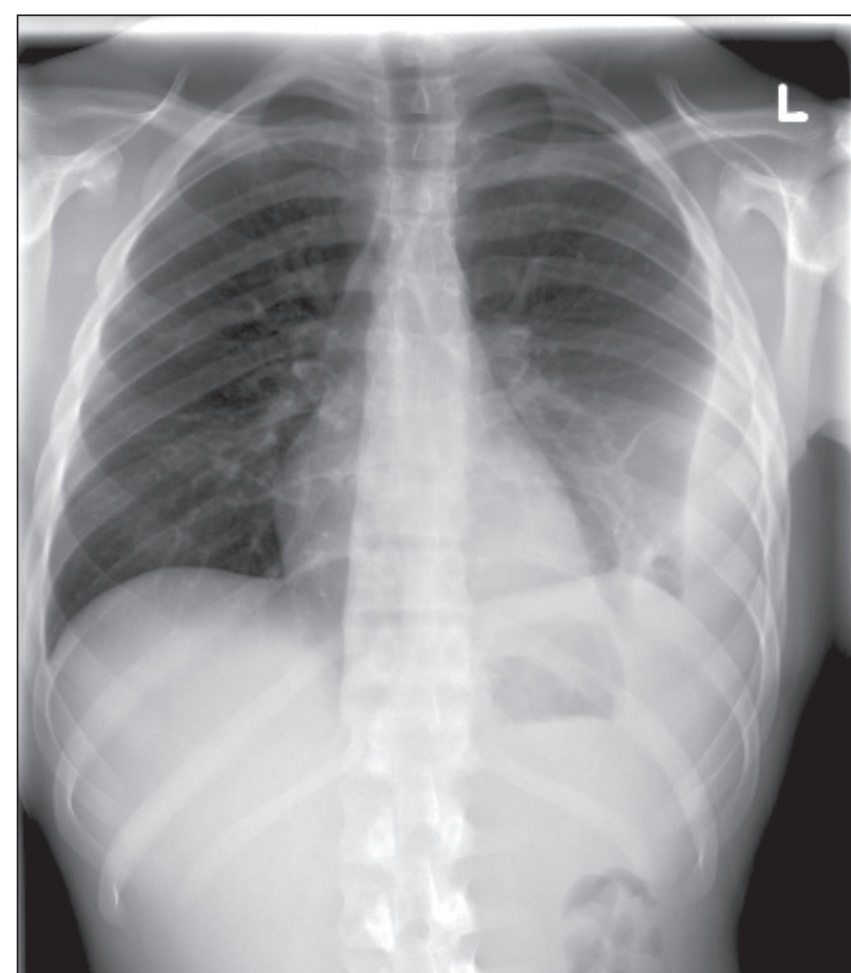

Figure 2) Chest radiograph showing a left-sided, loculated pleural effusion

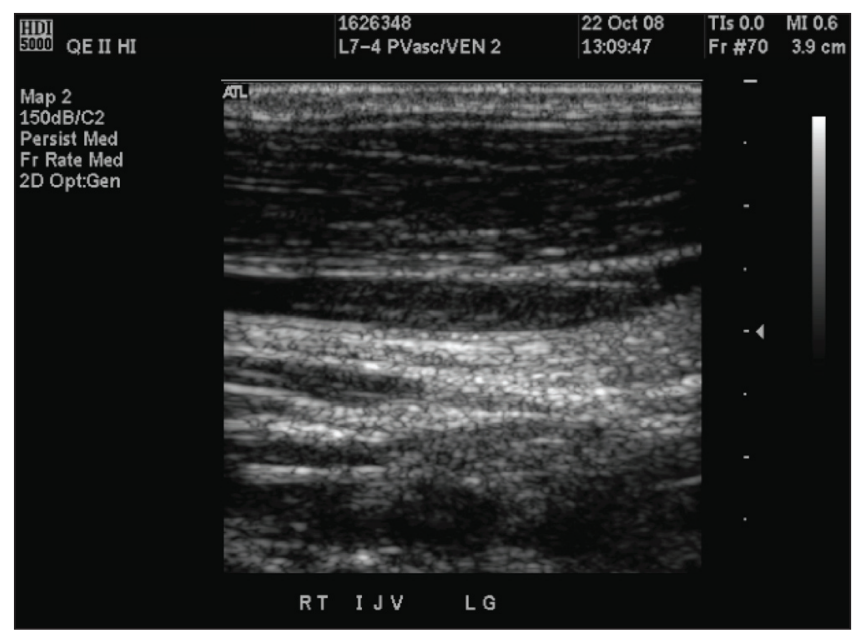

Figure 3) Doppler ultrasound of the right internal jugular vein. A nonocclusive thrombosis is seen within the lumen of the artery

\section{DISCUSSION}

Lemierre's disease is a clinical syndrome defined as oropharyngeal sepsis, thrombophlebitis of the IJV and septic embolic phenomenon. It is a disease with incidence rates ranging from one to 14.4 per million per year $(1,2)$. It is typically described in young, immunocompetent individuals, with a mean age of onset of 20 years.

Infection is described in phases. Phase one begins with pharyngeal infection. The initial site of infection is usually the palatine tonsils $(87.1 \%)$; however, seeding can also stem from odontogenic infections (1.8\%) or mastoiditis (2.7\%) (3). Phase two occurs with invasion into the lateral pharyngeal space, usually via the lymphatic system. The posterior lateral 
pharyngeal space contains neurovascular structures including the carotid artery, internal jugular vein, hypoglossal, glossopharyngeal and vagus nerves, and the cervical sympathetic trunk. IJV infection and thrombosis are the most common consequences. However, the IJV is rarely palpable, even when thrombotic (3). Neck pain and tenderness are common complaints; however, $47.7 \%$ of patients have no neck symptoms (3). Other neurovascular complications have been documented, including Horner's syndrome, paralysis of the trapezius muscle and carotid artery rupture (3).

Tertiary infection occurs when septic thromboemboli seed distant organs, with pleuropulmonary involvement being most common (92\%) (4). Septic arthritis was part of Lemierre's original diagnostic triad; currently, however, it is a less common complication (13\% to $27 \%$ ) (4). Abnormal liver function is encountered in almost one-half (49\%) of patients; however, liver and splenic abscesses are relatively rare $(4 \%$ and $3 \%)$. Skin involvement is rare (0\% to $3 \%)$, usually in the form of subcutaneous abscesses (4).

Blood cultures positive for Fusobacterium species should indicate the diagnosis. Fusobacterium are Gram-negative, nonsporeforming obligate anaerobes. They comprise part of the normal flora of the nasopharynx, gastrointestinal tract and female genital tract. The genus is unusual in its ability to produce devastating septic sequelae in immunocompetent hosts. Lemierre's disease is classically associated with a monoinfection of Fusobacterium species (87.5\%); however, polymicrobial and non-Fusobacterium Lemierre syndromes are documented (5).

\section{REFERENCES}

1. Ajulo P, Qayyum A, Brewis C, Innes A. Lemierre's syndrome: The link between a simple sore throat, sore neck, and pleuritic chest pain. Ann R Coll Surg Engl 2005:87:303-5.

2. Hagelskjaer Kristenesen L, Prag J. Lemierre's syndrome and other disseminated Fusobacterium necrophorum infections in Denmark: A prospective epidemiological and clinical survey. Eur J Clin Microbiol Infect Dis 2008;27:779-89.

3. Chirinos JA, Lichtstein DM, Garcia J, Tamariz LJ. The evolution of Lemierre's syndrome: Report of 2 cases and review of the literature. Medicine (Baltimore) 2002:81:458-65.

4. Ruirdan T. Human infection with Fusobacterium necrophorum (Necrobacillosis) with a focus of Lemierre's syndrome. Clin Microbiol Rev 2007;20:622-59.

5. Goldenberg N, Knapp-Clevenger R, Hays T, Manco-Johnson M. Lemierre's and Lemierre's-like syndromes in children: Survival and thromboembolic outcomes. Pediatrics 2005;116:543-8.

6. Appelbaum PC, Spangler SK, Jacobs MR. Beta lactamase production and susceptibilities to amoxicillin, amoxicillin-clavunate, ticacillin,
Diagnostic criteria for the syndrome are not well defined. It is clear that infections should arise from the oropharynx. It is not clear whether IJV thrombosis is a necessary criterion, or whether embolic phenomena such as septic pulmonary embolism or lung infarcts are appropriate surrogate markers. Documented cultures are not crucial for diagnosis (4).

Long-term antibiotics remain a cornerstone of treatment. Antibiotics should be tailored against Fusobacterium species. These microorganisms are usually resistant to gentamicin, quinolones and tetracycline. There is usually good sensitivity to penicillin; however, resistance has been documented (6-8). Metronidazole is an acceptable antibiotic choice, with good microbial coverage for Fusobacterium species, and appears to be the favoured antibiotic (9). The appropriate duration of treatment is not defined; however, at least six weeks is the generally accepted minimum (10).

Anticoagulation is often suggested as treatment; however, there is no evidence supporting its use. Anecdotally, it has been found to reduce septic foci and limit embolic showers. No randomized control trials have been conducted. Generally, anticoagulation is considered when cerebral infarct or cavernous sinus thrombosis is present $(4,10,11)$.

Our patient presented in renal failure, which has been reported previously (12). As well, the initial abnormal $\mathrm{x}$-ray (Figure 1) and the abdominal computed tomography examination (not shown) were suggestive of embolic disease. The latter is characteristic of Lemierre's syndrome, with the lung commonly involved $(3,4)$.

ticacillin-clavulanate, cefoxitin, imipenem and metronidazole of 320 non-Bacteroides fragilis bacteroides isolates and 120 fusobacteria from 28 US centres. Antimicrob Agents Chemother 1990;34:1546-50.

7. Moreno SJG, Altozano B, Pinilla J, et al. Lemierre's disease: Postanginal bacteremia and pulmonary involvement caused by Fusobacterium necrophorum. Rev Infect Dis 1989;11:319-24.

8. Ahkee S, Srinatha S, Huang A, Raff MJ, Ramirez JA. Lemierre's syndrome: Postanginal sepsis due to anaerobic oropharyngeal infection. Ann Otol Rhinol Laryngol 1994;103:208-10.

9. Armstrong, A, Spooner S, Sanders J. Lemierre's Syndrome. Curr Infect Dis Report 2000:2:168-73.

10. Bondy P, Grant T. Lemierre's syndrome: What are the roles for anticoagulation and long-term antibiotic therapy? Ann Otol Rhinol Laryngol 2008;117:679-83.

11. Hoehn KS. Lemierre's syndrome: The controversy of anticoagulation. Pediatrics 2005;115:1415-6.

12. Iwamoto Y, Kawakami H, Kishi F, Miyamoto M, Minakata T. Lemierre's syndrome with acute renal failure. Nippon Naika Gakki Zasshi 2007;96:2792-3. 


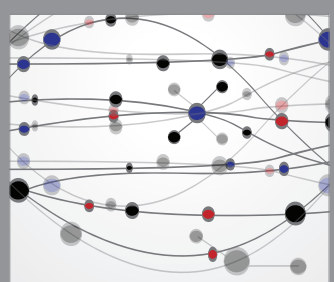

The Scientific World Journal
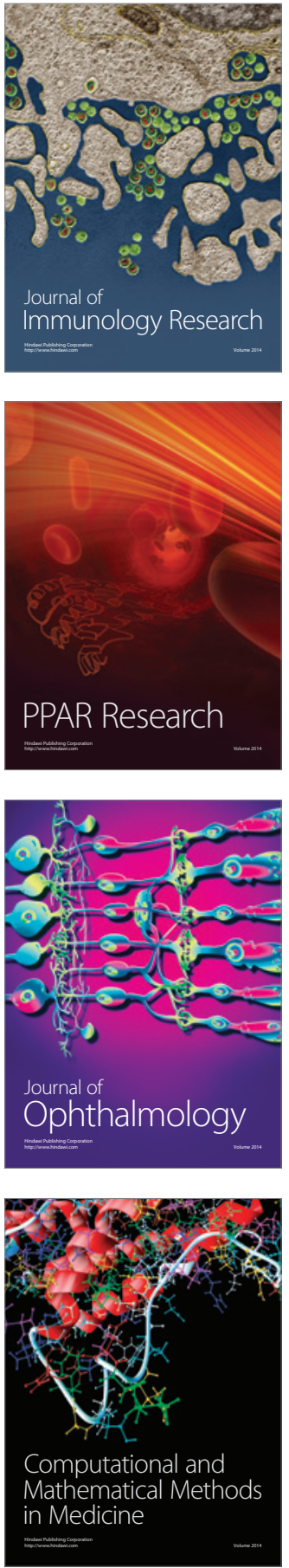

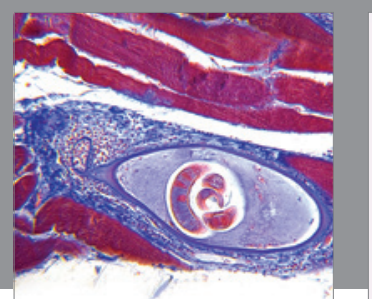

Gastroenterology Research and Practice

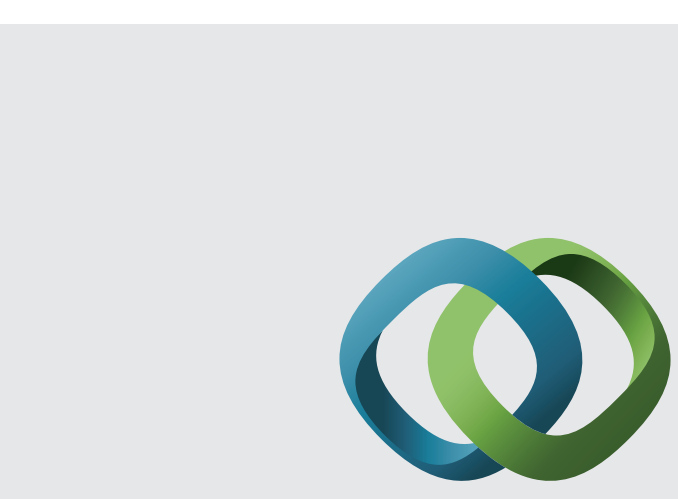

\section{Hindawi}

Submit your manuscripts at

http://www.hindawi.com
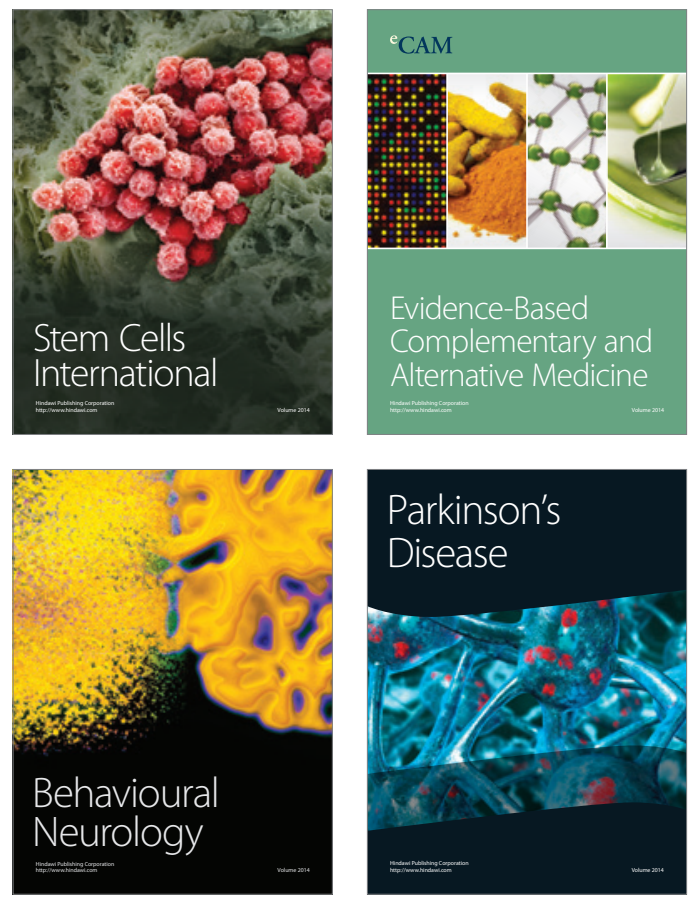
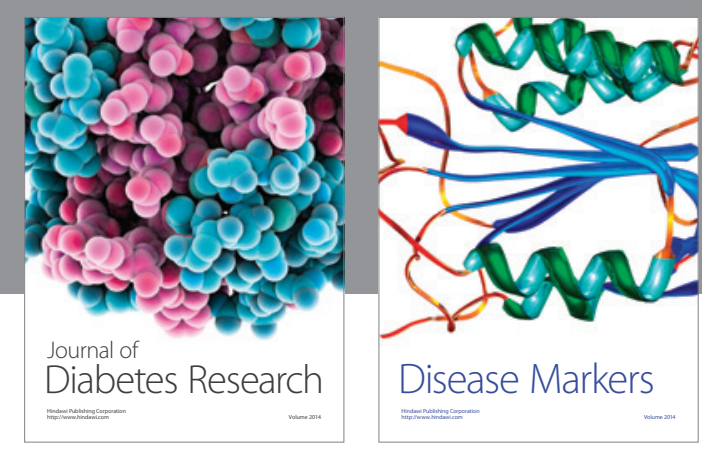

Disease Markers
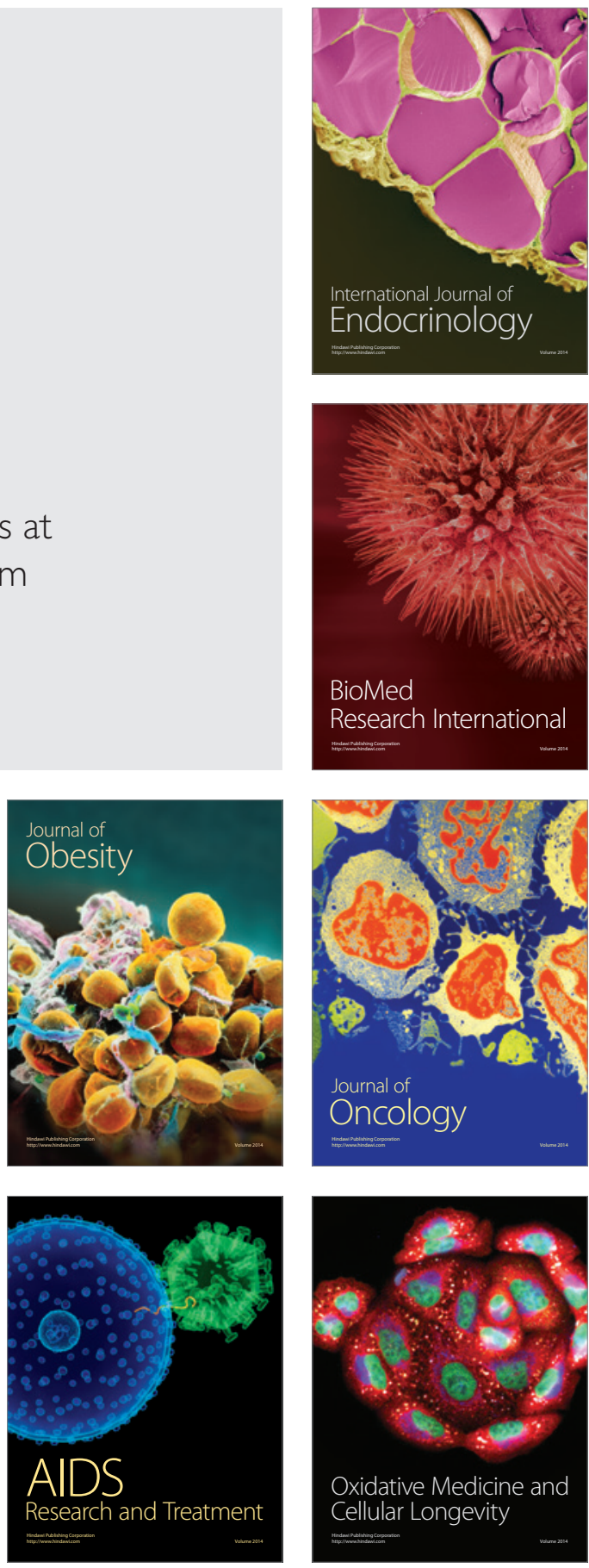